\title{
Lymphedema in palliative care - a case of reverse hypo- dermoclysis
}

\author{
Rebecca Haworth, MD', Robin Grant, MD², Erin Gorman-Corsten, MD² \\ I. Department of Family Medicine, McMaster University, Hamilton, Ontario, Canada. \\ 2. Division of Palliative Medicine, Dalhousie University and Nova Scotia Health Authority, Halifax, Nova Scotia, Canada.
}

\section{Case Summary}

This case demonstrates how the palliative procedure of reverse dermoclysis may be utilized to reduce lymphedema. Our patient had a brief trial of reverse dermoclysis, draining a total of $1700 \mathrm{~mL}$ of interstitial fluid from her lower legs. She reported improved mobility and quality of life.

\section{Background}

Lymphedema is common in patients nearing the end of life. Lymphedema is defined as an accumulation of fluid in the interstitial space and is caused by the dysfunction of normal flow between fluid spaces. ${ }^{1}$ Its cause is multifactorial, including increased arterial capillary leakage, decreased venous capillary reabsorption, or a change in the constitution of the fluid such as hypoproteinemia. ${ }^{1}$

Treatments for this debilitating condition can be divided into two main categories: medicinal and mechanical. The most common medications used to treat lymphedema include diuretics and corticosteroids. $^{2}$ Multiple mechanical approaches can be used to treat lymphedema including elevating the affected limb, manual lymphatic drainage, bandaging, exercise, and in specific situations, surgery, chemotherapy and radiotherapy. ${ }^{2}$ While these therapies, including compression bandaging, have been shown to be effective in treating lymphedema, the contribution of each individual therapy alone is not well understood. ${ }^{3}$ However, in palliative patients who are nearing end of life, these current treatments often fall short of decreasing the burden that lymphedema adds to their life.

With these patients in mind, the procedure of reverse hypodermoclysis (or reverse dermoclysis) was developed to increase quality of life by reducing lymphedema. It is an adaptation of the more common palliative procedure, hypodermoclysis. Hypodermoclysis is performed via insertion of a subcutaneous needle (butterfly, cannula) to slowly introduce fluid into the interstitial space. It allows for parenteral medications or small amounts of hydration to be delivered in a patient where intravenous access is not established. Conversely, reverse dermoclysis allows for removal of interstitial fluid.

Clein and Pugachev published a case series of eight patients in whom reverse dermoclysis was performed. ${ }^{4}$ The patients were predominantly male with significant edema of their legs and genitals, and it was suspected that several had partial obstruction of their inferior vena cavae, impairing lymphatic return. All of the patients had pelvic metastases, most were hypoalbuminemic, and three had hepatomegaly. Various techniques were used including making incisions, inserting penrose drains, and using subcutaneous butterfly sets. Seven of the eight patients drained a significant amount of fluid (1250-7000ml) and received meaningful symptom relief.

A more recent case series of two patients was published by Jacobsen and Blinderman. ${ }^{5}$ Although the techniques varied somewhat (use of six IV catheters for skin puncture, followed by drainage into an ostomy bag), these patients also reported improvement in quality of life and actually proceeded to repeat the procedure. Despite the success of reverse dermoclysis in these case series and report, there has been no subsequent research on the procedure.

\section{The Case}

Our patient was a 60-year-old female with colorectal cancer metastatic to liver. Significant bilateral pitting edema to above the knees was the primary barrier to her achieving meaningful quality of life in her final days. Her legs were so heavy she often required assistance lifting them onto her bed. This patient was no longer able to ambulate independently, which caused her great frustration. Prior to transfer to the palliative care unit, she had trialed furosemide (Lasix) with minimal effect. Tubigrip bandaging and compression stockings were tried, both causing discomfort with no appreciable reduction in her lymphedema. Reverse dermoclysis was considered with the goal of improving comfort and ambulation while minimizing cumbersomeness.

We reviewed the above case series and two Canadian hospital protocols. ${ }^{6,7}$ The procedure and potential risks of bleeding, ongoing drainage from needle sites, and infection were explained, and the patient provided informed consent. Her rationale was that she may experience improved mobility with the inconvenience of being attached to tubing and bags being a lesser evil. Our technique included the following steps:

- With the patient in bed, head elevated, legs on mattress, a subcutaneous butterfly with bevel up was inserted into the dorsum of each foot. 


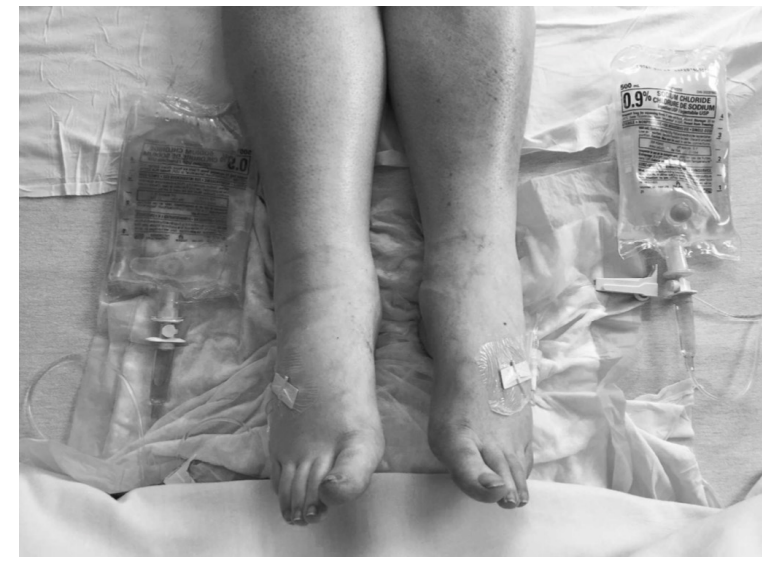

Figure I: Supplies in situ.

- Subcutaneous butterflies were secured with tegaderm adhesive.

- $500 \mathrm{~mL}$ saline bags (emptied) were attached to the butterflies and placed below the level of the feet.

This patient spent the majority of the ensuing 44 hours in bed, but ensured at least three hours per 24 hour period was in sitting position to allow gravity to assist drainage. After two hours, only one bag had fluid accumulating, so the subcutaneous butterfly was repositioned. Figure 1 shows the supplies in situ.

Figure 2 shows the patient's legs at 20 hours, at which time $900 \mathrm{~mL}$ of fluid had drained. As the patient perceived benefit at this time, she opted to continue for another 24 hours, during which time another $800 \mathrm{~mL}$ had drained (Figure 3). Both photos were taken immediately after the patient was asked to reposition herself from lying down to sitting on the edge of the bed.

In total, $1700 \mathrm{ml}$ of fluid had been drained from both legs over 44 hours. Our patient felt her legs were notably lighter, which improved her mobility. She felt the improvement was greater than what she received from compression bandages prior to attempting reverse dermoclysis. After the subcutaneous butterflies were removed, she did experience ongoing leakage which was controlled with absorbent dressings easily covered by socks, and she was able to ambulate outside. She felt the procedure did improve her quality of life and was content with the decision to try reverse dermoclysis.

\section{Discussion and Conclusion}

In this case, we used the palliative procedure of reverse dermoclysis to drain interstitial fluid from the legs of a patient who experienced significant lymphedema. Cost and burden for the patient are low, and it reportedly improved quality of life.

Practical considerations for our case would be using bags that can be hung on the bed / chair rather than resting on the floor and trying the bevel-down approach as suggested by the Waterloo protocol to

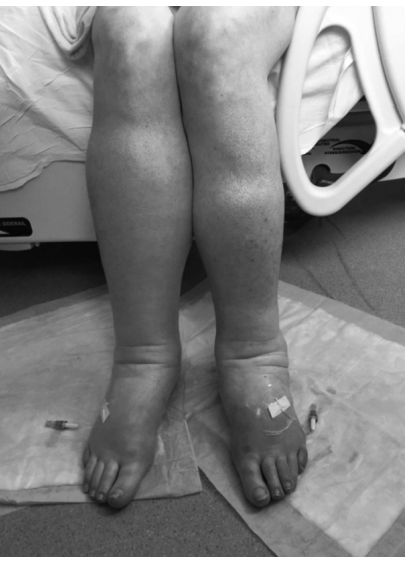

Figure 2: Drainage at 20 hours.

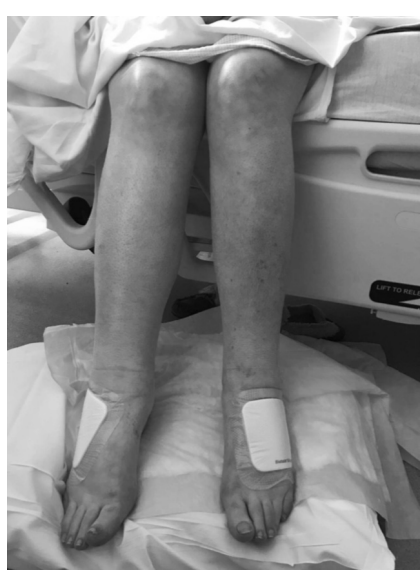

Figure 3: Drainage at 44 hours. theoretically improve drainage. ${ }^{5}$

Regarding potential for future research, several questions arise. Although there are protocols available for reverse dermoclysis, there is little data to guide patient selection. Similarly, there is little data suggesting what duration or frequency of procedure is most effectual. To our knowledge, there have been no trials comparing reverse dermoclysis to other common treatments such as positioning or bandaging.

Similar to the results reported by Clein and Pugachev, our trial of reverse dermoclysis was successful both objectively with significant fluid drainage and subjectively with improved mobility and comfort. ${ }^{3}$ We feel that this was a meaningful palliative care treatment that should be considered in other patients with burdensome lymphedema.

\section{Patient Consent}

Our patient provided verbal consent for the included photographs to be taken for teaching and education purposes. By the time that the case report was written, the patient had passed away, so written consent for the publication of this case report was provided by the patient's spouse.

\section{References}

1. Rockson, S. G. Lymphedema. The American journal of medicine, 2001;110(4):288-295.

2. Towers, A., Hodgson, P., Shay, C. and Keeley, V. Care of Palliative Patients with

3. Cancer-Related Lymphoedema. Journal of Lymphoedema, 2010:5(1):72-80.

4. Towers, A., Hodgson, P., Shay, C. and Keeley, V. Care of Palliative Patients with

5. Cancer-Related Lymphoedema. Journal of Lymphoedema, 2010:5(1):72-80.

6. Clein LJ, Pugachev E. Reduction of edema of lower extremities by subcutaneous controlled drainage: Eight cases. American
Journal of Hospice and Palliative Medicine, 2004; 21(3):228-232.

7. Jacobson, J \& Blinderman, C. Subcutaneous Lymphatic Drainage (Lymphcentesis) for Palliation of Severe Refractory Lymphedema in Cancer Patients. Journal of Pain and Symptom Management 2011;(41)6 1094-1097.

8. Koso, C., P\&P Subcutaneous Lower Extremity Lymphedema Drainage Procedure, Waterloo, ON. 2008

9. Frank, G, Wolch, G. Palliative Care Tips Issue \#36 Subcutaneous Drainage of Lower Limb Edema. Edmonton, AB. 2010 